\title{
Erlotinib after Failure of Gefitinib Treatment of More Than 6 Months in Advanced Non-Small Cell Lung Cancer
}

\author{
Kyoichi Kaira Nobuyuki Yamamoto \\ Division of Thoracic Oncology, Shizuoka Cancer Center, Shizuoka, Japan
}

Saito et al. [1] have documented in this issue of ONKOLOGIE that erlotinib is one of the therapeutic options for patients with advanced non-small cell lung cancer (NSCLC) with longterm stable disease (SD) of more than 6 months who had previously been on gefitinib. In their study, 21 patients with NSCLC were retrospectively evaluated, 9\% achieved a partial response (PR) and 36\% disease control (DC). The median survival time (MST) of patients with DC was 369 days, which was longer than in previous reports [2]. Their preliminary data suggest that erlotinib can produce clinical benefit in patients with long-term SD of more than 6 months on gefitinib treatment.

Recently, we described the pooled analysis of 11 published articles (106 patients) on erlotinib after failure of gefitinib [2]. With erlotinib therapy, PR was observed in $9.9 \%$ and DC in $29.2 \%$ of patients, corresponding to the results of Sato's study [1]. Cho et al. [3] also concluded that erlotinib seems to be a potential therapeutic option for the treatment of advanced NSCLC patients who show SD while receiving gefitinib. However, we could not clarify the mechanisms behind the effectiveness of erlotinib in this population. There are several potential explanations: (i) erlotinib was administered at the maximum tolerated dose, whereas gefitinib was administered at approximately one of third of its maximum tolerated dose. The standard doses of erlotinib and gefitinib are not biologically equivalent. (ii) The difference in tumor sensitivity might be associated with the relative concentration of epidermal growth factor receptor (EGFR) tyrosine kinase inhibitors (TKIs). (iii) The $\mathrm{IC}_{50}$ value of erlotinib is much lower than that of gefitinib [4].

On the other hand, Asami et al. [5] had documented that erlotinib may achieve a survival benefit for patients with pulmonary adenocarcinoma with less than 12 months of time to progression on prior gefitinib who showed a PR to gefitinib. A second biopsy was performed on the progressed lesions after failure of gefitinib therapy, and in cases with time to progression of less than 12 months no EGFR T790M mutations were found. Therefore, they suggest that tumor clones may not yet have acquired a secondary mutation in patients who have been receiving gefitinib for less than 12 months. Hara et al. [6] described that higher efficacy of erlotinib after gefitinib can achieved with optimal patient selection based on the following criteria: good performance status, benefit from prior gefitinib therapy, and insertion cytotoxic chemotherapies between gefitinib and erlotinib. In their study, 11 out of 125 patients had a PR and 44 patients had SD, yielding an overall response rate of $9 \%$ and a DC of $44 \%$. However, 32 patients with these optimal selection criteria had a response rate of $25 \%$ and a DC of $72 \%$. Wong et al. [7] reported on the efficacy as salvage treatment after failure of first-line gefitinib in NSCLC, and $18(85.7 \%)$ out of 21 patients had DC with gefitinib and $12(57.1 \%)$ patients with salvage erlotinib, demonstrating a significant association between DC with gefitinib and erlotinib $(p=0.031)$. They concluded that erlotinib was effective in NSCLC patients who had prior DC with first-line gefitinib. Song et al. [8] had documented that patients with without EGFR mutation can probably benefit from erlotinib treatment after gefitinib failure. The results of these studies suggest that the efficacy of erlotinib after failure of gefitinib is correlated with any benefit from prior gefitinib treatment, however the mechanisms have not been completely clarified.

Recently, Jackman et al. [9] proposed the following criteria for acquired resistance to EGFR-TKIs in NSCLC: (i) previous treatment with a single-agent EGFR-TKI; (ii) both or either of the following: (a) a tumor harboring an EGFR mutation known to be related to drug sensitivity or (b) objective clinical benefit by EGFR-TKI (PR or complete response, or significant (6 months) clinical benefit (SD) after initiation of EGFR-TKIs); (iii) disease progression while on continuous treatment with EGFR-TKIs within the last 30 days; and (iv) no intervening therapy between cessation of EGFR-TKIs and initiation of new therapy. The NSCLC patients with long-term SD of more than 6 months while receiving gefitinib also met these criteria, however, subsequent EGFR-TKI treatment has been reported to achieve clinical benefit. 12 studies regarding

\section{KARGER}

Fax +497614520714

Information@Karger.de

www.karger.com (c) 2012 S. Karger GmbH, Freiburg

0378-584X/12/0351-0008\$38.00/0

Accessible online at:

www.karger.com/onk
Kyoichi Kaira, MD

Division of Thoracic Oncology, Shizuoka Cancer Center

1007 Shimonagakubo Nagaizumi-cho Sunto-gun

Shizuoka, 411-8777, Japan

Tel. +81 55 989-5222, Fax -5634

kkaira1970@yahoo.co.jp 
erlotinib after failure of gefitinib have been published, however, 9 are retrospective and only 3 prospective. To evaluate the efficacy of erlotinib as salvage treatment after failure of prior gefitinib, a large prospective study is warranted to confirm these observations.

In summary, the findings of Saito and colleagues demonstrate the clinical efficacy of erlotinib in NSCLC patients with long-term SD of more than 6 months while receiving gefitinib. This retrospective study has a small sample size which may give rise to selection bias. Out of the NSCLC patients with significant benefit from prior gefitinib therapy, some may benefit from erlotinib as salvage treatment. Little is known about the detailed mechanisms of efficacy of erlotinib after failure of gefitinib. A significant (6 months) clinical benefit (SD) after initiation of EGFR-TKIs has been described to be one of the factors for acquired resistance to EGFR-TKIs in NSCLC, therefore, we do not recommend to routinely administer erlotinib to NSCLC patients with long-term SD of more than 6 months after gefitinib treatment.

\section{Disclosure Statement}

The authors have no financial or personal relationships with other people or organizations that could inappropriately influence their work.

\section{References}

1 Saito H, Murakami S, Kondo T, Oshita F, Noda K, Yamada K: Effectiveness of erlotinib in advanced non-small-cell lung cancers in cases of gefitinib resistance after treatment of more than 6 months. Onkologie 2012;35: DOI: 10.1159/000335736.

2 Kaira K, Naito T, Takahashi T, Ayabe E, Shimoyama R, Kaira R, Ono A, Igawa S, Shukuya T, Murakami H, Tsuya A, Nakamura Y, Endo, Yamamoto N: Pooled analysis of the reports of erlotinib after failure of gefitinib for non-small cell lung cancer. Lung Cancer 2010;68:99-104.

$\checkmark 3$ Cho BC, Im CK, Park MS, Kim SK, Chang J, Park JP, Chi HJ, Kim YJ, Shin SJ, Sohn JH, Kim H, Kim JH: Phase II study of erlotinib in advanced non-small-cell lung cancer after failure of gefitinib. J Clin Oncol 2007;25:2528-33.
4 Sharma SV, Bell DW, Settleman J, Haber DA: Epidermal growth factor receptor mutations in lung cancer. Nat Rev Cancer 2007;7:169-81.

5 Asami K, Kawahara M, Atagi S, Kawaguchi T, Okishio K: Duration of prior gefitinib treatment predicts survival potential in patients with lung adenocarcinoma receiving subsequent erlotinib. Lung Cancer 2011;73:211-6.

6 Hata A, Katakami N, Yoshioka H, Fujita S, Kunimasa K, Nanjo S, Otsuka K, Kaji R, Tomii K, Iwasaku M, Nishiyama A, Hayashi H, Morita S, Ishida T: Erlotinib after gefitinib failure in relapsed non-small cell lung cancer: Clinical benefit with optimal patient selection. Lung Cancer 2011;74:26873.
7 Wong MK, Lo AI, Lam B, Lam WK, IP MS, Ho JC: Erlotinib as salvage treatment after failure to first-line gefitinib in non-small cell lung cancer. Cancer Chemother Pharmacol 2010;65:1023-8.

8 Song ZB, Yu YF, Chen ZW, Lu S: Erlotinib as a salvage treatment for patients with advanced nonsmall cell lung cancer after failure of gefitinib treatment. Chin Med J 2011;24:2279-83.

9 Jackman D, Pao W, Riely GJ, Engelman JA, Kris MG, Jänne PA, Lynch T, Johnson BE, Miller VA: Clinical definition of acquired resistance to epidermal growth factor receptor tyrosine kinase inhibitors in non-small-cell lung cancer. J Clin Oncol 2010;28:357-60. 